\title{
Dipole giant resonances in deformed heavy nuclei
}

\author{
J. A. Maruhn, ${ }^{1,2}$ P. G. Reinhard, ${ }^{3,2, *}$ P. D. Stevenson, ${ }^{4,5,2}$ J. Rikovska Stone,,${ }^{5,6}$ and M. R. Strayer ${ }^{7}$ \\ ${ }^{1}$ Institut für Theoretische Physik, Universität Frankfurt, D-60325 Frankfurt, Germany \\ ${ }^{2}$ Joint Institute for Heavy Ion Research, Oak Ridge, Tennessee 37831, USA \\ ${ }^{3}$ Institut für Theoretische Physik II, Universität Erlangen, D-91058 Erlangen, Germany \\ ${ }^{4}$ Department of Physics, University of Surrey, Guildford, GU2 7XH, United Kingdom \\ ${ }^{5}$ Department of Physics, University of Oxford, Oxford, OX1 3PU, United Kingdom \\ ${ }^{6}$ Department of Chemistry and Biochemistry, University of Maryland, College Park, Maryland 20742, USA \\ ${ }^{7}$ Physics Division, Oak Ridge National Laboratory, Oak Ridge, Tennessee 37831-6373, USA
}

(Received 22 December 2004; published 30 June 2005)

\begin{abstract}
The spectral distribution of isovector dipole strength is computed using the time-dependent Skyrme-HartreeFock method with subsequent spectral analysis. The calculations are done without any imposed symmetry restriction, allowing any nuclear shape to be dealt with. The scheme is used to study the deformation dependence of giant resonances and its interplay with Landau fragmentation (owing to 1ph states). Results are shown for the chain of $\mathrm{Nd}$ isotopes, superdeformed ${ }^{152} \mathrm{Dy}$, triaxial ${ }^{188} \mathrm{Os}$, and ${ }^{238} \mathrm{U}$.
\end{abstract}

DOI: 10.1103/PhysRevC.71.064328

PACS number(s): 21.60.Jz, 24.30.Cz, 31.15.Ne

\section{INTRODUCTION}

The nuclear giant dipole resonance (GDR) is a most pronounced feature in the excitation spectrum, giving crucial clues to the understanding of nuclear structure and dynamics. Since its first observation [1], it has been much studied. For reviews see, for example [2,3] from the experimental side and [4-7] for the theoretical aspects, or [8] for both. Yet, there are still many interesting questions that deserve continued studies on that subject. The upcoming possibility to measure GDR in exotic nuclei [9] challenges theorists to scrutinize the predictive value of their models. Of particular interest here are self-consistent mean-field approaches that describe the ground-state and the excitation spectrum within one and the same parametrization. For a recent review see, for example [10]. The additional information from GDR probes otherwise loosely fixed aspects of effective mean-field models. This has been investigated for the case of the Skyrme-Hartree-Fock (SHF) method in, for example [11,12] and there is still some way to go for a full incorporation of all that information into effective mean-field theories. One such aspect that has not yet been much investigated is GDR in deformed nuclei, this is due to the great technical complications involved in the corresponding calculations. Nonetheless, deformed nuclei provide an interesting testing ground because there is a strong interplay between the structure of the GDR and the ground-state deformation [13].

It is the aim of this paper to present an exploration of the GDR in deformed nuclei, computing the excitation spectra using the SHF method. The deformation removes the degeneracy of the resonance peaks in spatial $x, y$, and $z$ directions, leading to a collective splitting of the resonance [13]. A competing mechanism that also spreads the resonance peak to some extent is Landau fragmentation, which results from a coupling of the resonance with energetically close

*Electronic address: mpt218@theorie2.physik.uni-erlangen.de one-particle-one-hole (1ph) states. (Landau fragmentation is the discrete-spectrum cousin of Landau damping, which is a well-known effect in bulk matter [14].) The interplay of these two mechanisms was studied recently for the case of deformed metal clusters and it was found that Landau fragmentation can mask deformation splitting under certain conditions [15]. We will address this question here for the case of medium-heavy and heavy nuclei.

A standard method to compute GDR spectra is the randomphase approximation (RPA) [5,7]. It can be considered as the small-amplitude limit of time-dependent mean-field equations $[16,17]$, but most practical solution schemes map that formally into an algebra of $1 \mathrm{ph}$ states. This becomes computationally very expensive for deformed systems owing to an enormous proliferation of necessary $1 \mathrm{ph}$ states. A formally simpler alternative is to use the straightforward time-dependent meanfield theory and extract the resonance pattern by spectral analysis of the time-dependent results. This method has been used successfully many times for computing resonance spectra in metal clusters [18-20]. It has also been applied in nuclear physics with symmetry restrictions [21-24], and more recently in some unrestricted calculations [25-27], which promise a rich future for the method. In this paper, we use a fully fledged time-dependent SHF model without any symmetry restrictions to compute the isovector-dipole strength in deformed nuclei. Only a brief outline of the model is given in Sec. II. Technical details of the method will be fully described in a forthcoming paper.

\section{FORMAL FRAMEWORK}

\section{A. Mean-field equations}

In the following, we give only a brief account of the timedependent SHF method and its solution. The underlying SHF functional and subsequent mean-field equations are explained in more detail in, for example $[10,28,29]$. 
The starting point is the SHF energy functional $E=$ $E(\rho, \tau, \mathcal{J}, \mathbf{j}, \sigma)$ expressed in terms of a few local densities and currents obtained as a sum over single-particle wave functions: density $\rho$, kinetic density $\tau$, spin-orbit density $\mathcal{J}$, current $\mathbf{j}$, and spin density $\sigma$, where each features twice, once for protons and once for neutrons. It is augmented by a pairing functional deduced from a zero-range two-body force (volume pairing). The mean-field equations are derived by variation with respect to the densities, yielding for the static case

$$
\hat{h} \varphi_{\alpha}=\varepsilon_{\alpha} \varphi_{\alpha}
$$

and for the time-dependent case

$$
\imath \partial_{t} \varphi_{\alpha}=\hat{h} \varphi_{\alpha}
$$

where $\varphi_{\alpha}$ are the active single-nucleon wave functions and $\hat{h}$ is the mean-field Hamiltonian, which depends on all densities and currents. This is complemented by the gap equations. We treat pairing within the BCS approximation [10] and we do that only for the static solution. The occupation amplitudes are then fixed during the time evolution of the system. This is an acceptable approximation for the spectra in the resonance region (i.e., for energies around and above $10 \mathrm{MeV}$ ). Dynamical changes in pairing will mostly affect the low-lying states not considered here.

The practical solution of the mean-field equations employs a representation of fields and wave functions on a three-dimensional Cartesian grid in coordinate space whereas derivatives are evaluated in Fourier-transformed space [30]. We use grids with $24 \times 24 \times 24$ points (the $z$ dimension is extended to 32 points for ${ }^{238} \mathrm{U}$ ) and a grid spacing of $1 \mathrm{fm}$. The fast Fourier transformation (FFT) is used for swapping between coordinate and momentum space. The accelerated gradient iteration is employed to find the stationary ground-state solution [30,31]. A Taylor series expansion of the mean-field propagator is used for the dynamical time stepping [32]. The size of the time step is $0.2 \mathrm{fm} / c$.

\section{B. The choice of the force}

The SHF functional sets only a framework within which there are myriad different parametrizations. Almost all parameter sets describe ground-state properties equally well but differ in other observables (e.g., excitation spectra or nuclear matter properties) [33]. One thus should, in principle, always consider several sufficiently different parametrizations to disentangle particularities of a given parametrization from general features of the SHF model. Large-scale surveys in connection with the GDR in spherical nuclei had been performed in $[11,12]$. The computationally demanding, fully triaxial calculations force us to constrain the variety. We use here the two parametrizations SkI3 [34] and SLy6 [35]. Both are recent developments that try to take care of special features in addition to the usual ground-state properties. SkI3 employs an extended spin-orbit functional whose isovector properties resemble relativistic models. SLy6 has a bias on neutron-rich systems up to neutron stars. Both provide a reasonable description of the GDR in ${ }^{208} \mathrm{~Pb}[10,12]$. As a brief reminder of typical results for GDR with SHF, we show
TABLE I. The average GDR resonance frequencies for two doubly magic nuclei computed with the two forces under consideration and compared with experimental (Exp.) data [3]. The dipole spectrum of ${ }^{16} \mathrm{O}$ is very fragmented. An average in the range $18-26 \mathrm{MeV}$ had been taken.

\begin{tabular}{lccc}
\hline \hline Nucleus & $\begin{array}{c}\text { Exp. } \\
(\mathrm{MeV})\end{array}$ & $\begin{array}{c}\text { SLy6 } \\
(\mathrm{MeV})\end{array}$ & $\begin{array}{c}\text { SkI3 } \\
(\mathrm{MeV})\end{array}$ \\
\hline${ }^{16} \mathrm{O}$ & 23.5 & 19.6 & 20.2 \\
${ }^{208} \mathrm{~Pb}$ & 13.5 & 13.9 & 13.4 \\
\hline \hline
\end{tabular}

results in Table I for one very light and one heavy doubly magic nucleus for the two forces. The results are typical for all other presently available Skyrme forces [12]. One can achieve a good description of the GDR position in ${ }^{208} \mathrm{~Pb}$ and at the same time one grossly underestimates the resonant range for ${ }^{16} \mathrm{O}$ where the average resonance strength lies $2-4 \mathrm{MeV}$ below the experimental data. We thus constrain consideration to heavier systems where we hope that we can trust the given parametrizations with respect to GDR.

\section{Spectral analysis}

The dipole strength of the nuclei is computed using the standard procedure of spectral analysis [19]. We start from the ground state and apply an instantaneous momentum boost to the center of mass of the protons with a reverse boost to the neutrons. This means that each nucleon wave function is augmented by a phase factor

$$
\varphi_{\alpha}(\mathbf{r}) \longrightarrow \varphi_{\alpha}(\mathbf{r}, t=0)=e^{\imath \mathbf{b}_{v} \mathbf{r}} \varphi_{\alpha}(\mathbf{r}),
$$

where $\mathbf{b}_{v}$ is the boost momentum of nucleon type $v$. Proton and neutron boost sum to conserve center-of-mass momentum (i.e., $Z \mathbf{b}_{p}+N \mathbf{b}_{n}=0$ ). The amplitude of the boost is kept sufficiently small to stay well in the linear regime of excitations. The direction of the boost selects the mode that is excited. We chose a diagonal excitation with $\mathbf{b}_{v} \propto$ $\mathbf{e}_{x}+\mathbf{e}_{y}+\mathbf{e}_{z}$ to access all three basic modes at once. The three components of the total isovector-dipole momentum are recorded during the subsequent dynamics, yielding the dipole signal $\mathbf{D}(t)$ in the time domain. Spectral analysis requires some filtering to avoid artefacts from the limited time span of computation [36]. (We compute up to a time of $1600 \mathrm{fm} / c$ which leaves sufficient resolution for the GDR region.) We do filtering in the time domain using a Gaussian smoothing $D(t) \longrightarrow D(t) \exp \left[-(t / \tau)^{2}\right]$ with $\tau=350 \mathrm{fm} / c$. Finally, the dipole strength (=ptical absorption strength) is obtained as the imaginary part of the Fourier transform [i.e., $S_{i}(\omega)=$ $\left.\Im\left\{\tilde{D}_{i}(\omega)\right\}\right]$. Having the three spatial components recorded separately, we obtain separate information about the dipole strength for all three modes. The simplification of using a diagonal excitation is applicable if the optical axes of the nucleus are identical with the axes of the coordinate system. All considered nuclei fulfill this condition because their ground state is reflection symmetric with respect to all three axes. To make the smoothing effective we run the dynamics up to at least $4 \tau=1400 \mathrm{fm} / c$. The spectral resolution is then approximately 
$\Delta \omega \approx 4 \tau^{-1} \approx 2 \mathrm{MeV}$. This suffices for studies in the GDR region. The GDR peak is in the particle continuum. We use a finite box that artificially discretizes the continuum. The resolution of $2 \mathrm{MeV}$ is larger than the expected escape width such that this sort of continuum effect is masked anyway.

\section{RESULTS AND DISCUSSION}

\section{A. Simple estimates}

There is a very simple, and yet reliable, estimate for the average frequency of the GDR peak [37]:

$$
\omega_{0}=80 \mathrm{MeV}^{-1 / 3} \text {. }
$$

Note the trend $\propto A^{-1 / 3} \propto R^{-1}$, where $R$ is the nuclear radius. This is motivated by the viewpoint that the GDR is zero-sound and that the largest wavelength in a finite system is proportional to $R$. The estimate (4) is a useful guideline for spherical nuclei. Deformed nuclei show a splitting that can be estimated by considering the trend with inverse length for each direction separately, thus $\omega_{i} \propto R_{i}^{-1}$ for $i \in\{x, y, z\}$. The deformation can be described by stretching factors $R_{i} \longrightarrow \eta_{i} R$, where $\eta_{x} \eta_{y} \eta_{z}=1$. This yields immediately $\omega_{i}=\omega_{0} / \eta_{i}$. For axially symmetric systems, one has $\eta_{x}=\eta_{y}$, which leaves one number to characterize the deformation. Let us assume for instance that this is done in terms of the axis ratio

$$
\zeta=R_{z} / R_{x}=\eta_{z} / \eta_{x} .
$$

Together with the condition $\eta_{x}^{2} \eta_{z}=1$, we can then easily derive the simple estimate

$$
\omega_{x, y}=\omega_{0} \zeta^{1 / 2}, \quad \omega_{z}=\omega_{0} \zeta^{-1} .
$$

More often, deformation is characterized in terms of the quadrupole momentum $Q_{20}=\left\langle r^{2} Y_{20}\right\rangle$. This, however, changes scale with $R^{2}$ and thus hinders an immediate geometrical appreciation. Therefore, one prefers the dimensionless quadrupole momentum

$$
\beta_{2 \mu}=\frac{4 \pi}{5} \frac{\left\langle r^{2} Y_{2 \mu}\right\rangle}{A r_{0}^{2}}, \quad r_{0}=0.93 A^{1 / 3},
$$

where only $\mu=0$ and $\mu=2$ are needed in a properly aligned (i.e., intrinsic) system. Note that the denominator is often taken as $\left\langle r^{2}\right\rangle$ instead of the $A r_{0}^{2}$. The two conventions are practically identical for small deformations. The present choice is advantageous for large deformations where it stretches the scale. For axially symmetric systems, one encounters $\beta_{22}=0$. The relation of $\beta_{20}$ to the axis ratio (5) is then for small deformations

$$
\zeta \approx 1+\frac{3}{2} \sqrt{\frac{4 \pi}{5}} \beta_{20},
$$

with obvious consequences for the estimated GDR frequencies (6). Figure 1 sketches a schematic GDR splitting along the three principle axes of an axially symmetric nucleus. The peak position of each of the collective dipole modes is indicated by an arrow. The relative strengths indicate the sign of deformation. Prolate nuclei have two states at the higher frequency.

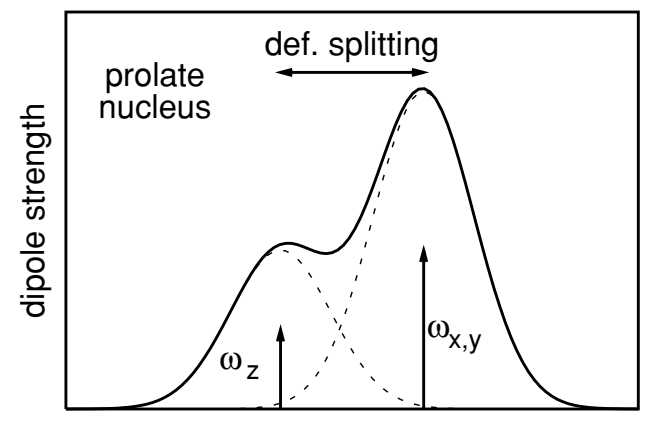

$\omega$

FIG. 1. Schematic view of collective dipole strengths in a prolate deformed nucleus. The dashed lines show the strength for the $x-y$ and $z$ modes separately.

The lines are broadened by several effects (for an extensive review see [38]): coupling of the collective strength to the $1 \mathrm{ph}$ states (spreading width, often called Landau fragmentation), particle escape into the continuum (escape width; see, e.g. [39]), correlations from nucleon-nucleon collisions (collisional broadening, see, e.g. [40]), and coupling to low-lying vibrations (phonon coupling; see, e.g. [41]). The last two broadening mechanisms are related to $2 \mathrm{ph}$ or higher configurations and go beyond a mean-field treatment. The collisional correlations deliver a smooth broadening of about 0.5-1 MeV. The surface coupling depends sensitively on the softness of the nucleus, being small for near-magic or well-deformed nuclei and larger in the transitional region. Neither of these effects are accounted for in the present calculations. We assume that they are hidden under the rather large smoothing width of about $2 \mathrm{MeV}$. The Landau fragmentation and escape width belong to $1 \mathrm{ph}$ mechanisms and are accessible to mean-field models. The escape width is related to the particle continuum, giving each single-particle state in the energy range of 5-10 MeV a width of 0.5-1 Mev. Such a splitting is not resolved with the present precision. Thus three of the broadening mechanisms-collisional, phonon coupling, and escape-are masked by the global width introduced through smoothing the signal. This is indicated by the broadening in Fig. 1. The collective splitting can be nicely seen provided that its energetic separation exceeds the line broadening and that the broadening is smooth.

There remains the Landau fragmentation, which is the major source of width in nuclei. The density of 1 ph states in medium-size nuclei is not so large, and thus Landau fragmentation can induce a discrete pattern rather than a global broadening. The effects depend on the detailed shell structure of a given nucleus and even change for different forces. Figure 2 shows the dipole spectrum in doubly magic ${ }^{132} \mathrm{Sn}$ in high resolution to display more clearly the distribution of dipole strength over the range of the GDR. The spectrum in the GDR region shows very detailed structures that are related to the underlying spectrum of $1 \mathrm{ph}$ states. (A detailed discussion for the comparable case of metal clusters is found in [42].) An almost direct relation between the small peaks in the wings of the dipole distribution and the $1 \mathrm{ph}$ states can be established when taking into account that the residual interaction causes a 


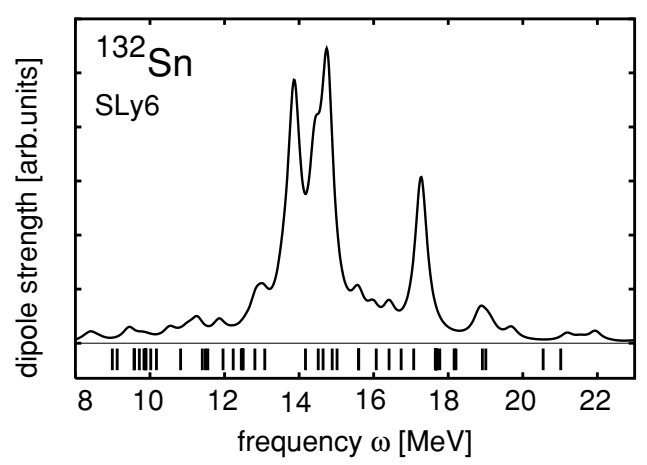

FIG. 2. Demonstration of Landau fragmentation with a highly resolved dipole spectrum for ${ }^{132} \mathrm{Sn}$ computed with SLy6. The full line shows the GDR spectrum. The dashes underneath indicate the positions of the individual $1 \mathrm{ph}$ with nonvanishing dipole strength.

small downshift for the low-lying states and an upshift at the high-lying side. Experimentally, the high resolution in Fig. 2 is unrealistic because the other aforementioned broadening mechanisms smooth the spectrum. In practice, one sees a broad GDR peak with some structures where the fragmentation is larger than the smoothing. For example, the well-separated large peak at about $17 \mathrm{MeV}$ will show up as a high-energy shoulder in the spectrum. As a general rule, the collective resonance [see Eq. (4)] as well as the typical 1ph transitions follow the same trend $\propto A^{-1 / 3}$. Thus Landau fragmentation is equally important in all nuclei. However, the spectral density increases with $A$ such that the broadening becomes smoother for heavy nuclei whereas very fuzzy distributions are seen for light nuclei (and also for exotic ones). The nuclei that are studied here are mostly of medium size. It is interesting to see how the fragmentation performs there. As a side remark, we note that metal clusters are finite fermion systems, which have many similarities with nuclei [43]. They also have a giant dipole resonance, called the Mie surface plasmon. And again, this mode shows significant Landau fragmentation in the range of medium sizes [15] whereas the pattern becomes much smoother for huge sizes $(N \approx 1000$, a size not accessible to nuclei) [44].

Since both ways of expressing deformations are in use, we provide in Table II the information on deformation for all axially symmetric test cases together with the deduced estimates for the peak frequencies. According to our SHF calculations, we have one fully triaxial nucleus in the sample, ${ }^{188} \mathrm{Os}$. Its axes are related as $z: y: x=1.62: 1.22: 1$, yielding the estimated frequencies $\omega_{x}=17.46 \mathrm{MeV}, \omega_{y}=14.25 \mathrm{MeV}$, and $\omega_{z}=10.74 \mathrm{MeV}$. The superdeformed state in ${ }^{152} \mathrm{Dy}$ is a matter of debate. It has been found also in other mean-field calculations [45-47], but its appearance depends on the force and pairing recipe. We take it here as a principle example for a superdeformed state.

\section{B. Transition to deformation}

Nuclei in the region $Z \approx 60$ show a transition from spherical, at the neutron closure $N=82$, to deformed nuclei when $N$ is safely above 82 . We consider here as one typical
TABLE II. The axis ratios $\zeta$, dimensionless quadrupole deformations $\beta_{20}$, and estimated GDR peak frequencies for the axially symmetric test case nuclei. All results are computed with SLy6, except for ${ }^{238} \mathrm{U}$ where SkI3 was used. The axis ratios are deduced from the rms extensions in $x$ and $z$.

\begin{tabular}{lcccc}
\hline \hline Nucleus & $\zeta$ & $\beta_{20}$ & $\omega_{x}(\mathrm{MeV})$ & $\omega_{z}(\mathrm{MeV})$ \\
\hline${ }^{142} \mathrm{Nd}$ & 1.00 & 0.00 & 15.30 & 15.30 \\
${ }^{144} \mathrm{Nd}$ & 1.05 & 0.03 & 15.64 & 14.90 \\
${ }^{146} \mathrm{Nd}$ & 1.15 & 0.08 & 16.29 & 13.21 \\
${ }^{148} \mathrm{Nd}$ & 1.21 & 0.11 & 16.64 & 12.50 \\
${ }^{150} \mathrm{Nd}$ & 1.30 & 0.17 & 17.17 & 11.58 \\
${ }^{152} \mathrm{Dy}_{\text {obl }}$ & 0.87 & -0.13 & 13.98 & 17.23 \\
${ }^{152} \mathrm{Dy}_{\text {prol }}$ & 1.15 & 0.15 & 16.06 & 13.04 \\
${ }^{152} \mathrm{Dy}_{\text {supdef }}$ & 1.86 & 0.90 & 20.44 & 8.06 \\
${ }^{238} \mathrm{U}$ & 1.25 & 0.28 & 14.43 & 10.33 \\
\hline \hline
\end{tabular}

example the chain of $\mathrm{Nd}$ isotopes. Figure 3 shows dipole strengths for the separate modes and in total as computed with SkI3. The arrows indicate the estimated mean positions of the $x, y$, and $z$ modes (see, Sec. III A). They are degenerate as they should be for a spherical nucleus. The spectrum, however, shows strong fragmentation with a well-developed side peak.

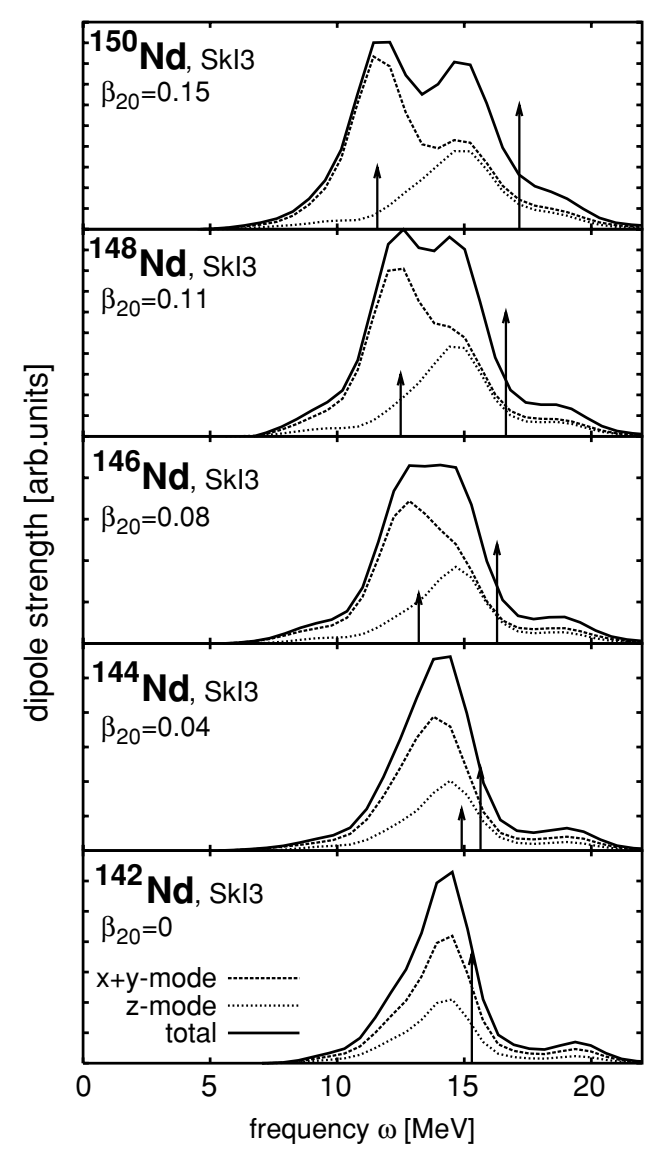

FIG. 3. Dipole strengths in the chain of $\mathrm{Nd}$ isotopes computed with SkI3: Dashed lines denote modes along $x$ and $y$ directions, dotted lines denote mode along $z$ direction, and solid lines denote the total computed strength. The deformation is indicated in each panel. The estimated peak positions (see Sec. III A) are indicated by arrows. 
This is due to a redistribution of strength by coupling to energetically close $1 \mathrm{ph}$ states (Landau fragmentation). The effect should, in principle, deliver a smooth peak broadening and it would do so in much heavier systems. Nuclei around $A \approx 150$ still have obviously a too dilute $1 \mathrm{ph}$ spectrum, which, in turn, can yield a structured peak broadening. The estimate (4) with (6), although a bit too high, shows satisfying agreement in view of its enormous simplicity.

The deformation grows gradually when moving from ${ }^{142} \mathrm{Nd}$ to ${ }^{150} \mathrm{Nd}$. The separate strengths show the corresponding collective shifts more or less in agreement with the simple estimates. It is obvious, however, that in the transitional region the width of the peaks is larger than the shift. This inhibits a clear identification of weak deformations, particularly because Landau fragmentation reduces the apparent peaks and can give misleading visual impressions. It is only for the heaviest and most strongly deformed nucleus in the sample that deformation splitting safely outweighs Landau fragmentation. The collective estimate (6) shows a splitting of the same order, however always with a tendency to overestimate. There is a further feature by which the detailed results differ from the schematic view of Fig. 1. One rarely spots the weight of the mode from the mere height of the peaks. Broadening can be much different in the two modes and one has to analyze the total strength under a peak. This goes beyond a simple graphical inspection and requires peak fitting. Altogether, the analysis of deformation is somewhat uncertain because of the interplay with Landau fragmentation. This sets an uncertainty of $\delta \beta \approx 0.05$ in the analysis provided it is done on the grounds of comparison with microscopic calculations. Deducing deformation from scanning two peaks and relating it to the simple estimate (6) adds another bit of systematic error. These findings are very similar to the results for medium-size metal clusters [15].

Figure 4 compares the results from two different Skyrme forces with experimental data. In all cases the average strength fits nicely to the experimental data with a tendency to a slight downshift of order $1 \mathrm{MeV}$. The agreement is better for ${ }^{208} \mathrm{~Pb}$ and much worse for light nuclei (see Table I). It is not yet clear whether the underestimation of about $1 \mathrm{MeV}$ seen here is already the beginning of the trend.

The theoretical spectra in the spherical ${ }^{142} \mathrm{Nd}$ show strong Landau fragmentation for both forces. The details, however, differ owing to a somewhat different shell structure. The high-lying shoulder is farther away from the resonance for SkI3 and is consequently weaker. Unfortunately, it seems that the experimental resolution does not allow sufficient detail in the fragmentation to be discriminated, or it may be that there is less fragmentation than both Skyrme forces show. The deformation is growing slowly with increasing $N$. The next nucleus in the chain, ${ }^{144} \mathrm{Nd}$, is still dominated by Landau fragmentation. But a weak deformation splitting may be seen already for ${ }^{146} \mathrm{Nd}$ for SkI3 where the $1 \mathrm{ph}$ splitting is higher up in the spectrum whereas the more compact pattern of SLy6 still hides the collective effect. The experimental data are, however, too coarse to resolve any detail here. A similar situation is found for ${ }^{148} \mathrm{Nd}$. In fact, the qualitative differences between SkI3 and SLy6 look even more dramatic here. The overall profile of the peak with a slight asymmetry toward low frequencies

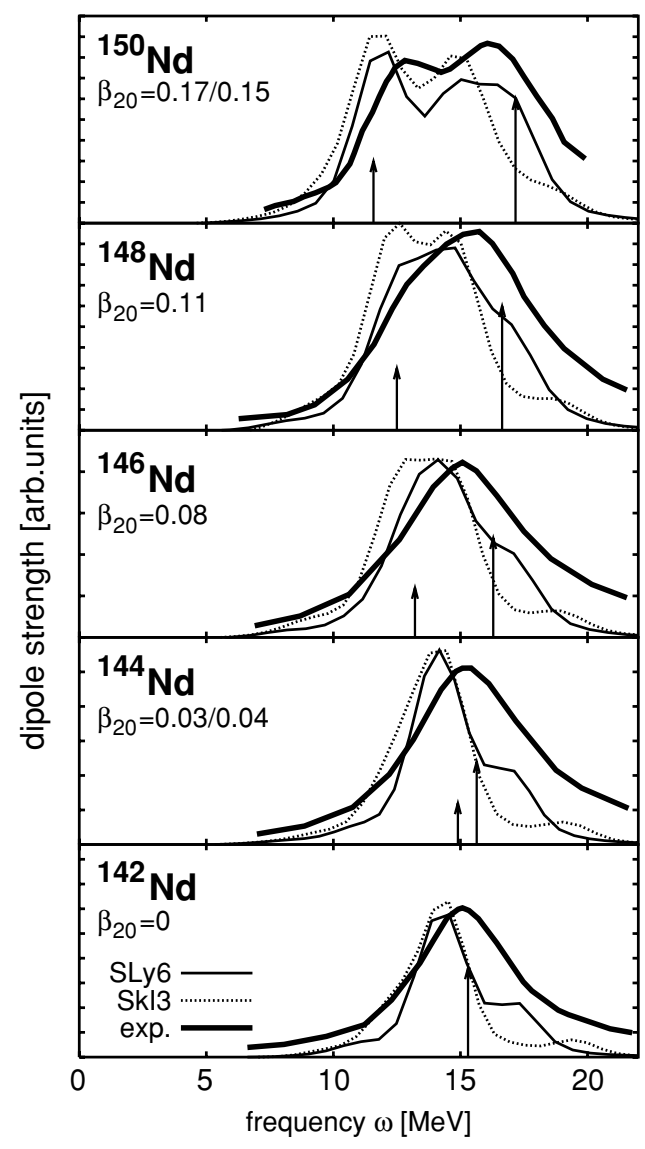

FIG. 4. Total dipole strengths in the chain of $\mathrm{Nd}$ isotopes computed with SkI3 as well as with SLy6 and compared with experimental data from [2]. The deformation is indicated in each panel. The first value is associated with SLy6 for ${ }^{144} \mathrm{Nd}$ where two values are shown. The estimated peak positions (see Sec. III A) are indicated by arrows.

seems to be better fitted by SLy6. Finally, the largest nucleus in the sample, ${ }^{150} \mathrm{Nd}$, is sufficiently well deformed to show qualitatively clear signs of deformation splittings in equal manner for both forces and in the data. Even the magnitude of splitting seems to match. The slightly smaller splitting produced by SkI3 may be closer to the experimental situation, but this observation has to be taken with care because the results are masked to some extent by Landau fragmentation.

\section{A superdeformed isomer}

We have learned in the previous subsection that one needs a sufficiently large deformation to make the deformation splitting visible above the "background" of Landau fragmentation and other broadening effects. It is natural then to go one step further and to look for a superdeformed nucleus. Figure 5 shows the case of ${ }^{152}$ Dy as one example. The energy surface in the upper panel shows that there is a prolate ground state as well as an oblate and a superdeformed isomer. The lower panels show the spectra for all three minima. The prolate and the oblate states show spectra where deformation splitting and Landau fragmentation are strongly entangled. 

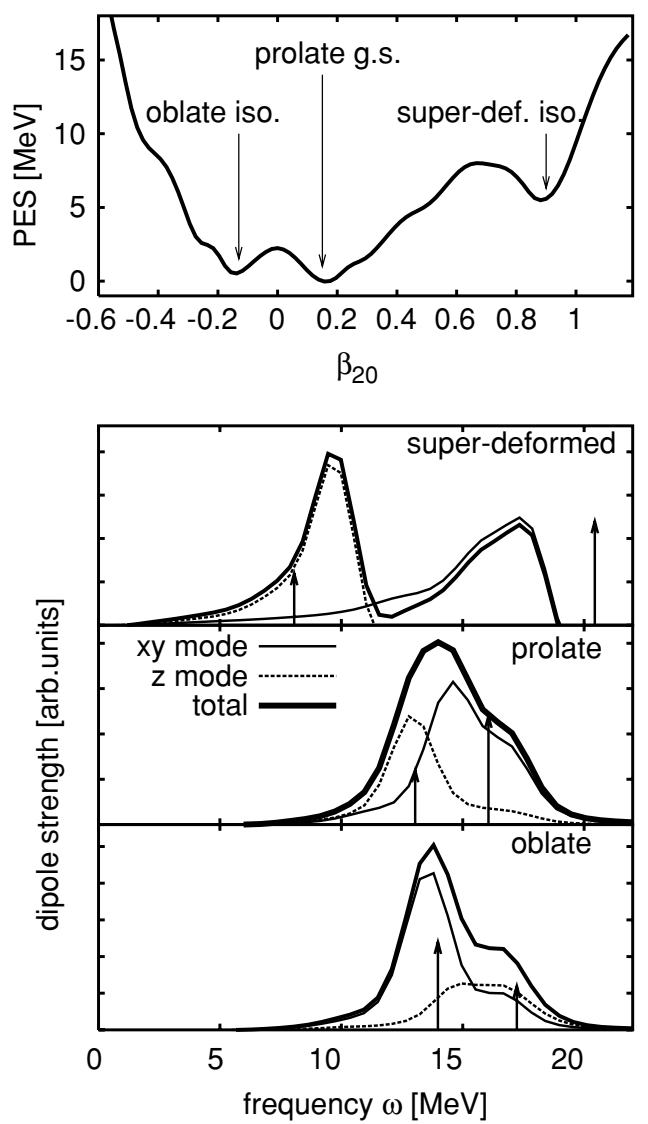

FIG. 5. (Upper panel) Quadrupole deformation energy surface for ${ }^{152}$ Dy computed with SLy6. (Lower panels) Dipole strengths in ground state and isomers as shown in the upper panel. The full lines denote modes along $x$ and $y$ directions, the dotted lines denote the mode along the $z$ direction, the heavy solid lines denote the total strength. The estimated peak positions (see Sec. III A) are indicated by arrows.

The entanglement is worse than for ${ }^{150} \mathrm{Nd}$, where the splitting was better separated despite the fact that both systems have about the same deformation. This shows clearly that a careful microscopic analysis is required before concluding that there is a deformation splitting from an experimental GDR spectrum. The superdeformed state, however, indicates very clear splitting, larger than the line widths, which allows an immediate identification of deformation. Similar structures were found previously in a calculations based on a deformed shell model [48]. A word of caution is in place here. The resonance spectrum has been computed in the limit of smallamplitude oscillations about the local minimum. A very small amplitude, corresponding to an average excitation energy of about $0.1 \mathrm{MeV}$, has been used to stay safely in the vicinity of the minimum. The actual excitation energy, however, is at least $8 \mathrm{MeV}$ and thus far above the barrier to the ground state. The photo-excited nucleus will thus move quickly toward the ground-state shape, which, in turn, broadens the resonance substantially. A full description of this process requires a very elaborate mixing of collective quadrupole motion with the giant resonance [49], which goes beyond the scope of this paper. The simple estimate (6) is producing a splitting that

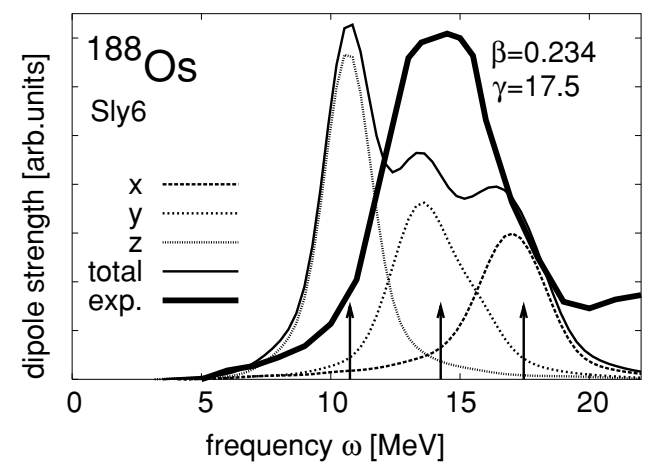

FIG. 6. Dipole strength in the triaxial ground state of ${ }^{188} \mathrm{Os}$ computed with SLy6. The total strength is shown as well as the strengths for the separate modes. The experimental result from [3] is shown for comparison. The estimated peak positions (see Sec. III A) are indicated by arrows. $\beta=\sqrt{\sum_{\mu} \beta_{2 \mu}}$ is the total quadrupole deformation and $\gamma=\operatorname{atan} \sqrt{2} \beta_{22} / \beta_{20}$ is a measure of the triaxiality.

is somewhat too large. This is not surprising for this large deformation because the simple geometrical idea behind the estimate (simple ellipsoidal shapes) may be too crude. Keeping that in mind, a $30 \%$ precision of an empirical formula is a nice achievement.

\section{Triaxiality}

Triaxial nuclei display three different principal axes and thus also deliver three different GDR peaks, as discussed in Sec. III A. Deformation splitting is distributed over an additional peak and thus is probably harder to identify. One needs a nucleus with a sufficiently large net deformation. Our calculations provided such a situation for ${ }^{188}$ Os. Figure 6 shows its dipole strength distribution. Somewhat to our surprise, the perturbing effects from Landau fragmentation are small here and we resolve nicely a triple splitting in the theoretical calculation. However, the experimental data look much different in every respect. They do not show any indication of a triple splitting. The nucleus ${ }^{188}$ Os has an energy landscape with various isomers. Moreover, this landscape varies with various Skyrme forces to the extent that ground state and isomers are placed differently. It is very likely that the theoretical calculation for that particular force SLy6 has interchanged ground state with isomer. The figure then shows, at least from the theoretical side, the type of spectra at which one has to look to single out candidates for triaxiality.

\section{E. A heavy deformed nucleus}

Finally, it is worth checking a much heavier nucleus. Figure 7 shows the spectra for the ground state of ${ }^{238} \mathrm{U}$. In both modes, there is some shoulder from Landau fragmentation. However, the dominant peaks are sufficiently well developed to allow a discrimination of the deformation splitting. Both modes show a shoulder at about $15 \mathrm{MeV}$. The results agree fairly well with the experimental distribution concerning 


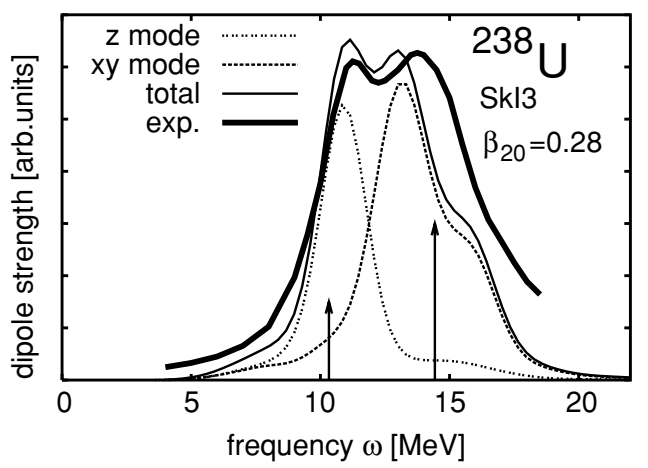

FIG. 7. Dipole strength in the prolate ground state of ${ }^{238} \mathrm{U}$ computed with SkI3. The total strength is shown as well as the strengths for the separate modes. The experimental result from [3] is shown for comparison. The estimated peak positions (see Sec. III A) are indicated by arrows.

overall position and splitting. They differ in detail to the extent that the data do not show such a clear shoulder.

\section{CONCLUSION}

We have computed dipole strength distributions for deformed nuclei within the framework of time-dependent Skyrme-Hartree-Fock method. The spectra are obtained from fully fledged dynamical calculations starting from an instantaneous isovector-dipole boost and subsequent spectral analysis of the emerging dipole signal. No symmetry restrictions are imposed. Thus all possible nuclear shapes can be tackled.

The scheme has been used to study, at a microscopic level, the deformation effects on the spectra in the region of the giant dipole resonance. Collective estimates predict a splitting of the GDR, where the mode vibrating along the compressed direction is shifted upword in frequency, whereas the elongated mode is shifted downword. The microscopic calculations confirm that effect, in principle. In practice, there is a competing mechanism that produces also detailed substructures in the spectra. This is Landau fragmentation caused by coupling of the collective strength to $1 \mathrm{ph}$ states that are close in energy. This effect is found to be still quite large for medium-heavy nuclei such that it masks the deformation splitting to a certain extent. Small deformations in the transitional region cannot be identified unambiguously from the GDR spectra alone. Well-deformed nuclei have a better chance to display the deformation splitting above the "background" of Landau fragmentation. We have even found visible signals of triaxial deformations. A particularly dramatic splitting, beyond any doubt, is found in superdeformed isomers.

The present exploration has employed only two different Skyrme forces to get some indication of the force sensitivity of the effects. The deformation splitting is found to be robust, but the detailed pattern from Landau fragmentation is more force dependent. More systematic surveys are needed to track the variance of theoretical results and to thus deliver reliable guidelines for the analysis of the data. Work in that direction is in progress.

\section{ACKNOWLEDGMENTS}

We thank Vladimir Pronyaev for help in collecting the experimental data. This work was supported in part by the Bundesministerium für Bildung und Forschung (BMBF), Project Nos. 06 ER 808 and 06 ER 124, by the US DOE Grant No. DE-FG02-94ER40834, and by EPSRC under Grant No. GR/S96425/01.
[1] W. Bothe and W. Gentner, Z. Phys. 71, 236 (1937).

[2] B. L. Bermann and S. C. Fultz, Rev. Mod. Phys. 47, 713 (1975).

[3] S. S. Dietrich and B. L. Bermann, At. Data Nucl. Data Tables 38, 199 (1988).

[4] O. Bohigas, A. M. Lane, and J. Martorell, Phys. Rep. 51, 267 (1979).

[5] K. Goeke and J. Speth, Annu. Rev. Nucl. Part. Sci. 32, 65 (1982).

[6] E. Lipparini and S. Stringari, Phys. Rep. 175, 103 (1989).

[7] G. F. Bertsch and R. Broglia, Oscillations in Finite Quantum Systems (Cambridge University Press, Cambridge, England, 1994).

[8] M. N. Harakeh and A. van der Woude, Giant Resonances (Oxford University Press, Oxford, 2001).

[9] A. Leistenschneider et al., Phys. Rev. Lett. 86, 5442 (2001).

[10] M. Bender, P.-H. Heenen, and P.-G. Reinhard, Rev. Mod. Phys. 75, 121 (2003).

[11] G. Colo, N. V. Giai, and H. Sagawa, Phys. Lett. B363, 5 (1995).

[12] P.-G. Reinhard, Nucl. Phys. A649, 305c (1999).

[13] M. Danos, Nucl. Phys. 5, 23 (1958).

[14] E. M. Lifschitz and L. P. Pitajewski, Physikalische Kinetik,
Vol. X of Lehrbuch der Theoretischen Physik (Mir, Moscow, 1988).

[15] V. O. Nesterenko, W. Kleinig, and P.-G. Reinhard, Eur. Phys. J. D 19, 57 (2002).

[16] D. J. Rowe, Nuclear Collective Motion (Methuen, London, 1970).

[17] G. E. Brown, Unified Theory of Nuclear Models and Forces (North-Holland, Amsterdam, 1971), 3rd ed.

[18] K. Yabana and G. F. Bertsch, Phys. Rev. B 54, 4484 (1996).

[19] F. Calvayrac, P.-G. Reinhard, and E. Suraud, Ann. Phys. (NY) 255, 125 (1997).

[20] P.-G. Reinhard and E. Suraud, Introduction to Cluster Dynamics (Wiley, New York, 2003).

[21] J. B. Ocki and H. Flocard, Phys. Lett. B85, 163 (1979).

[22] A. S. Umar and M. R. Strayer, Phys. Lett. B171, 353 (1986).

[23] S. Stringari and D. Vautherin, Phys. Lett. B88, 1 (1979).

[24] P. Chomaz and N. V. Giai, Phys. Lett. B189, 375 (1987).

[25] M. V. C. R. Chinn, A. S. Umar, and M. R. Strayer, Phys. Rep. 264, 107 (1996).

[26] T. Nakatsukasa and K. Yabana, Prog. Theor. Phys. Suppl. 146, 447 (2002). 
[27] C. Simenel and P. Chomaz, Phys. Rev. C 68, 024302 (2003).

[28] P.-G. Reinhard, Ann. Phys. (Leipzig) 1, 632 (1992).

[29] V. O. Nesterenko, J. Kvasil, and P.-G. Reinhard, Phys. Rev. C 66, 044307 (2002).

[30] V. Blum, G. Lauritsch, J. Maruhn, and P.-G. Reinhard, J. Comput. Phys. 100, 364 (1992).

[31] P.-G. Reinhard, in Computational Nuclear Physics I-Nuclear Structure, edited by K. Langanke, S. Koonin, and J. Maruhn (Springer, Berlin, 1991), p. 28.

[32] H. Flocard, S. E. Koonin, and M. S. Weiss, Phys. Rev. C 17, 1682 (1978).

[33] J. R. Stone, J. C. Miller, R. Koncewicz, P. D. Stevenson, and M. R. Strayer, Phys. Rev. C 68, 034324 (2003).

[34] P.-G. Reinhard and H. Flocard, Nucl. Phys. A584, 467 (1995).

[35] E. Chabanat, P. Bonche, P. Haensel, J. Meyer, and R. Schaeffer, Nucl. Phys. A635, 231 (1998); A643, 441(E).

[36] W. H. Press, S. A. Teukolsky, W. T. Vetterling, and B. P. Flannery, Numerical Recipes (Cambridge University Press, Cambridge, England, 1992).
[37] P. Ring and P. Schuck, The Nuclear Many-Body Problem (Springer-Verlag, New York, 1980).

[38] G. F. Bertsch, P. F. Bortignon, and R. A. Broglia, Rev. Mod. Phys. 55, 287 (1983).

[39] M. Cavinato, M. Marangoni, P. Ottaviani, and A. Saruis, Nucl. Phys. A373, 445 (1982).

[40] P.-G. Reinhard, C. Toepffer, and H. L. Yadav, Nucl. Phys. A458, 301 (1986).

[41] G. Colo and P. F. Bortignon, Nucl. Phys. A687, 282c (2001).

[42] P.-G. Reinhard, O. Genzken, and M. Brack, Ann. Phys. (Leipzig) 5, 1 (1996).

[43] P.-G. Reinhard, S. Weisgerber, O. Genzken, and M. Brack, Z. Phys. A 349, 219 (1994).

[44] J. Babst and P.-G. Reinhard, Z. Phys. D 42, 209 (1997).

[45] M. Girod, J. P. Delaroche, D. Gogny, and J. F. Berger, Phys. Rev. Lett. 62, 2452 (1989).

[46] I. Hamamoto and X. Z. Zhang, Phys. Rev. C 52, 2326(R) (1995).

[47] G. A. Lalazissis and P. Ring, Phys. Lett. B427, 225 (1998).

[48] Y. S. Chen, Phys. Rev. C 43, 173 (1991).

[49] P.-G. Reinhard and D. Drechsel, Nucl. Phys. A295, 125 (1978). 